\title{
Impact of Garlic-infused Salt Supplement on Fly Abundance, Salt Intake, and Defensive Behaviors in Grazing Beef Cows
}

\author{
Obioha Durunna ${ }^{1,2} \&$ Herbert Lardner ${ }^{2}$ \\ ${ }^{1}$ Department of Applied Research, Lakeland College, Vermilion, Alberta, T9X 1K5, Canada \\ ${ }^{2}$ Department of Animal and Poultry Science, University of Saskatchewan, Saskatoon, Saskatchewan, S7N 5A8, \\ Canada
}

Correspondence: Obioha Durunna, Department of Applied Research, Lakeland College, Vermilion, Alberta, T9X 1K5, Canada.

Received: November 4, 2020

Accepted: December 8, 2020 Online Published: December 24, 2020

doi:10.5539/sar.v10n1p54

URL: https://doi.org/10.5539/sar.v10n1p54

\begin{abstract}
This study evaluated the efficacy of free-choice feeding of garlic-infused salt as an alternative pest-fly control strategy in grazing beef cows. This on-farm study is relevant because pest-fly abundance during the summer can impact performance and may cause welfare concerns on beef cattle operations. Over two consecutive summers, three groups of grazing cows received either a trace-mineral salt (TMS) supplement or a TMS infused with garlic powder (GPTMS). The study assessed the treatment effects on fly abundance, defensive behavior, and salt consumption for each group. All cows grazed similar forage species. Fly abundance and defensive behaviors were evaluated in each group using digital imagery. The results showed no difference $(P=0.34)$ in monthly salt consumption among groups. There were differences $(P<0.0004)$ in fly abundance among groups where one GPTMS group had at least $47 \%$ fewer flies and $41 \%$ fewer defensive behaviors than the TMS groups. On the other hand, another group that received GPTMS in the second year did not differ $(P=0.07)$ in fly abundance when compared to its previous outcome when the group received the TMS in the first year. These differing results for fly abundance and defensive behavior in the groups that received the GPTMS suggest that the efficacy of this feeding strategy in cattle may be affected by differences in genetic or environmental factors or both. We recommended that future studies consider genomic breed compositions before assigning subjects to different groups. Additional studies are required to investigate the variation in individual supplement intake between treatment groups and whether infusing mineral supplements with garlic powder is associated with similar outcomes as salt.
\end{abstract}

Keywords: cattle, cow-calf, garlic powder, grazing, pest-flies, salt

\section{Introduction}

Summer grazing supports the cattle industry's competitiveness through low-cost body gains from pasture grazing (Mathis et al., 2008). However, the bites and irritations caused by summer pest flies, such as horn fly (Haematobia irritans irritans), face fly (Musca autumnalis), or stable fly (Stomoxys calcitrans), can have direct negative effects on cattle performance and behavior. These would result in interrupted grazing behavior, reduced growth performance, or transmission of diseases. Horn and stable flies are potent mechanical vectors of various diseases such as anthrax, vesicular stomatitis, and pink eye. Animals infested with high fly numbers that exceed economic thresholds could have 3-18\% reduced body weight (Campbell, 1976; Haufe, 1982) or 20\% reduced body gain (Campbell et al., 1987). Reports have also shown that a horn fly can consume about 11-21 mg of blood per day (Parra et al., 2013), impacting weight-gain and milk production. These undesirable impacts eventually reduce the bottom line for beef cattle operations. Therefore, controlling pest flies during the summer will improve animal productivity and profitability by improving animal health and growth performance.

The popularity of feeding supplements infused with garlic powder to cattle (as a fly-control option) during the summer is increasing among Canadian cattle producers, especially in the cow-calf sector. Although several fly-control options exist, their cost, practicality, social or public perception, and acceptance could be challenging. For example, while the use of synthetic repellents or insecticides could be economical and practical, there may be concerns about their unwanted impacts on human health and the development of pesticide resistance in the 
flies (Hashim and Davi, 2003; Meena et al., 2006). Feeding garlic-infused supplements are not only assumed to be economical under a free-choice feeding system but are purported to stimulate supplement consumption, especially for herds in regions where soils and forages are deficient in some critical nutrients. Because adequate intake of essential nutrients is necessary for improved growth and reproductive performance, supplementation is required in such regions. Therefore, improving mineral intake using effective supplementation strategies could improve cattle productivity. Besides, the idea that garlic powder is less likely to induce antimicrobial resistance (Karuppiah and Rajaram, 2012) would be more appealing to producers and the public, thereby enhancing the beef industry's social license. However, the scientific information on the efficacy of these garlic-laced supplements under real grazing environments is sparse.

Commercial garlic products are marketed as either dehydrated garlic powder or garlic oil extracted through steam distillation and are typically mixed with salt or mineral supplements. While these products' fly-repellence modus operandi in cattle is poorly understood, it is hypothesized that the repellence outcomes are due to actions of volatile sulfur-based compounds that are emitted from the skin after animals have ingested garlic-infused supplements. Therefore, this study's goal was to provide on-farm objective assessments of the insectifugal properties of garlic-infused trace mineral salt fed to grazing cows.

\section{Materials and Methods}

\subsection{Study Location and Animal Characteristics}

The study recruited three cow groups DM, GO, and RH (acronyms for identification purposes only), for an on-farm supplementation trial at the Beacon Hill Community Pasture, SK, Canada (54 $21^{\circ} 16.86$ N, $109^{\circ}$ $33^{\prime} 09.64 \mathrm{~W}$ ) in 2016 and 2017. The location had historical challenges with pest flies, especially horn flies. The cows were Simmental $\times$ Angus, or Simmental $\times$ Hereford $\times$ Angus crosses, and all three groups were managed in independent side-by-side pastures secured within barbed wire fences. All cows were cared for under standards comparable with the guidelines established by the Canadian Council on Animal Care guidelines (CCAC, 2009). Major forage species in the pastures were Kentucky bluegrass (Poa pratensis), meadow bromegrass (Bromus riparius Rehm.), and smooth bromegrass (Bromus inermus Leyss.). The supplement comprised either the trace mineral salt (TMS; Compass Minerals, Overland Park, Kansas) or TMS mixed with a commercial food-grade dehydrated garlic powder (GPTMS; Long Wing International Inc., Burlington, ON, Canada).

\subsection{Research Design and Data Collection}

The 2-year study followed a randomized research design where each cow-group received either a GPTMS or a TMS. The GPTMS fed in 2016 contained about 2\% garlic powder, while the GPTMS provided in 2017 had 5\% garlic powder. The bioactive compounds in the garlic powder samples were not measured. All supplements were fed free choice in three mineral tubs (for each group) located close to drinking sites to ensure easy access and intake. A crossover design was not used because the objective was to track the supplementation strategy's impact throughout the summer from an applied research perspective. Further, implementing a crossover design was not practical given the short fly season, limited information on the appropriate washout period, and the potential carry-over effects from the supplements.

The first year of the project ran from May 25 to September 13, 2016, while the second year ran from May 29 to September 14, 2017. In Year 1, the DM group received GPTMS (DM-G, 155 cows) while the RH (RH-N, 119 cows) and GO (GO-N, 157 cows) received TMS. In Year 2, the study maintained the DM group on GPTMS (DM-G, 176 cows), the RH group also received the GPTMS (RH-G, 116 cows), while GO remained on TMS (GO-N, 183 cows). Digital video footage and images of the cows' lateral body sections (for fly abundance and defensive behaviors) were collected during the survey sessions using a Sony® High Definition Handycam ${ }^{\circledR}$ camcorder. The surveys were conducted between 10:00 and 15:00 h on each survey day for all groups. This timeframe was maintained throughout the trial, but survey activities that were not accommodated within this timeframe (due to inclement weather conditions or other factors) and were not included in data analyses. The duration of each session was about 45 minutes in each group, in random sequences. The defensive behaviors evaluated during footage reviews were side-licks, tail-flicks, head-throws, leg-stomps, and belly-kicks (Mohammed et al., 2016). The number of animals exhibiting these behaviors as a proportion of all cows within the particular footage was calculated. The mineral tubs were refilled as needed with pre-weighed $25 \mathrm{~kg}$ bags. The supplement refills indicated the supplement disappearance (consumption) within each group.

The differences in fly abundance and defensive behaviors among the treatment groups were analyzed as repeated measures in SAS (SAS Institute Inc., Cary, NC) using a generalized linear mixed model (GLIMMIX Procedure). The model included the cow group (nested within treatment), and survey date were assumed as random effects. Within the GLIMMIX procedure, a negative binomial distribution was implemented for the fly abundance, while 
the proportion of animals exhibiting defensive behaviors involved a Beta distribution. A fixed-effects model for fly abundance, which included year, treatment, and yearxtreatment interaction, was also explored. The MIXED procedure of SAS was used to analyze the differences in monthly salt-supplement disappearance. Multiple comparisons of the treatment groups were computed with LSMEANS statement, while $P$-values from statistically significant effects were adjusted for the multiple comparisons using the Tukey-Kramer method.

\subsection{Statistics and Data Analysis}

Still-images of the lateral sections of surveyed cows were extracted from each footage using Adobe Premiere (Adobe Systems Incorporated, San Jose, CA). Statistical analyses of fly abundance (Table 1) included only red-hided cows because coat color may contribute to variation in fly abundance. There were cows with other coat colors, but they were not uniformly represented among the treatment groups. The majority of these flies rested on the crest-shoulder-rib sections of cows, suggesting that they were mostly horn flies. All resting flies in each image were manually counted by the same person using Adobe Photoshop CS6 (Adobe Systems Incorporated, San Jose, CA) software. In surveys that captured both sides of the same cow, the side with greater fly abundance was included in the analyses.

Table 1. Number of cows sampled over two years ${ }^{1}$

\begin{tabular}{llllllll}
\hline \multicolumn{3}{l}{ 2016 } & & & \multicolumn{3}{c}{ 2017 } \\
\hline Date & DM-G & GO-N & RH-N & Date & DM-G & RH-G & GO-N \\
\hline June 1 & 12 & 5 & - & June 7 & 38 & 16 & 25 \\
- & - & - & - & June 23 & 60 & 25 & 17 \\
July 6 & 8 & 13 & - & July 7 & 44 & 48 & 43 \\
- & - & - & - & July 13 & 60 & 20 & 27 \\
July 21 & 18 & 12 & 20 & July 28 & 36 & 12 & 20 \\
Aug 10 & 28 & 12 & 11 & Aug 17 & 31 & 26 & 14 \\
Aug 30 & 32 & 13 & 9 & Aug 30 & 33 & 6 & 18 \\
Sept 13 & 12 & 7 & 6 & Sept 14 & 23 & 11 & 21 \\
\hline
\end{tabular}

${ }^{1} \mathrm{DM}-\mathrm{G}$ received garlic powder-infused trace-mineral salt in both years; GO-N received only trace-mineral salt in both years; RH-N received only trace-mineral salt in the first year, while RH-G was switched to the garlic powder-infused trace-mineral salt supplements in the second year.

\section{Results}

Figure 1 shows the average fly abundance during the survey dates. Differences among the treatment groups for average fly abundance, defensive behaviors, and the monthly supplement disappearance are shown in Table 2 . There was a treatment effect $(P<0.0001)$ for fly abundance, where the DM-G group had over $47 \%$ fewer flies compared to the GO-N $(P<0.0001)$ and RH-N $(P<0.0001)$. The RH-G cow group also differed in fly abundance when compared to the GO-N $(P=0.03)$ but did not differ from the RH-N group $(P=0.07)$. Implementing a fixed-effects model that included year, treatment, and yearxtreatment interaction showed a significant year $\times$ treatment interaction $(P=0.0003)$ besides treatment $(P<0.0001)$ and year $(P=0.11)$ effects. The average number of flies for the DM-G group was not different $(P=0.96)$ between the two years, suggesting no differences between the efficacies of the inclusion rates (of garlic powder) in both years. Further, GO-N in both years did not differ $(P=0.17)$.

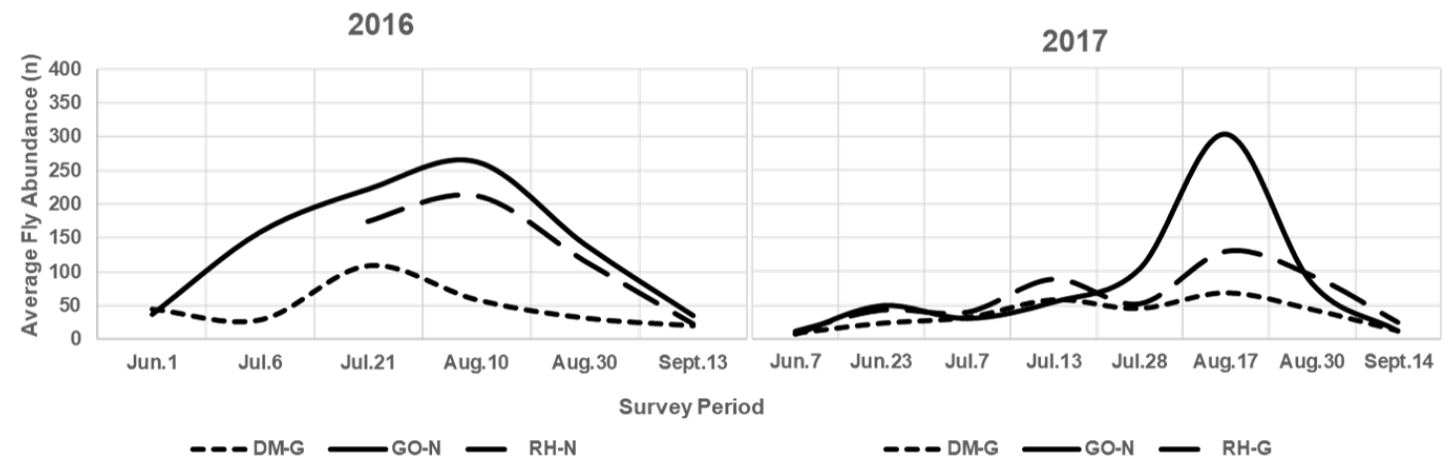

Figure 1. Fly abundance in both years 
DM-G received garlic-infused trace-mineral salt in both years; GO-N received only trace-mineral salt in both years. RH-N received only trace-mineral salt in the first year, while RH-G was switched to the garlic-infused trace-mineral salt supplements in the second year

Table 2. LSM $( \pm \mathrm{SE})$ for monthly supplement disappearance, fly abundance, and defensive behavior in the different animal groups ${ }^{1}$

\begin{tabular}{llllll}
\hline Factor & DM-G & RH-G & GO-N & RH-N & $\boldsymbol{P}$-value \\
\hline Total fly abundance (n) & $34.42 \pm 2.13^{\mathrm{a}}$ & $59.18 \pm 4.90^{\mathrm{b}}$ & $65.94 \pm 5.37^{\mathrm{c}}$ & $72.83 \pm 12.37^{\mathrm{bc}}$ & $<0.0001$ \\
2016 & 42.16 & - & 131.59 & 101.81 & \\
2017 & 29.70 & 47.10 & 44.75 & - & \\
Defensive behavior $^{2}, \%$ & $27.17 \pm 1.70^{\mathrm{a}}$ & $36.84 \pm 2.68^{\mathrm{b}}$ & $47.85 \pm 2.21^{\mathrm{c}}$ & $46.43 \pm 5.27^{\mathrm{bc}}$ & $<0.0001$ \\
Average salt intake $^{3}, \mathrm{~kg} / \mathrm{hd} /$ month & $1.25 \pm 0.23$ & $1.22 \pm 0.39$ & $1.57 \pm 0.22$ & $1.66 \pm 0.38$ & 0.65 \\
\hline
\end{tabular}

Within a row, different letters indicate significant differences between treatment groups $(P<0.05)$

${ }^{1}$ DM-G received garlic-infused trace-mineral salt in both years; GO-N received only trace-mineral salt in both years. RH-N received only trace-mineral salt in the first year, while RH-G was switched to the garlic-infused trace-mineral salt supplements in the second year. Fly abundance shows the number of flies counted on each animal.

${ }^{2}$ Defensive behavior shows the proportion of animals exhibiting side-licks, tail-flicks, head-throws, leg-stomps, and belly-kicks.

${ }^{3}$ Monthly supplement disappearance indicates supplement consumption by the different groups.

There were differences $(P<0.0001)$ among the treatment groups for defensive behaviors where DM-G had $42 \%$ fewer $(P<0.003)$ observed defensive behaviors than those that received salt-only. In comparison, RH-G group had 23\% fewer than the GO-N (Table 2) group. The defensive behaviors exhibited by RH-G cows were not different $(P=0.41)$ from the RH-N cows. The proportion of animals exhibiting defensive behavior did not differ $(P=0.99)$ between the GO-N and RH-N (Table 2).

The monthly average supplement disappearance (indicative of supplement consumption) in Table 2 showed no difference $(P=0.34)$ among the treatment groups. On average, the groups that received GPTMS consumed 41 $\mathrm{g} / \mathrm{d}$ of the salt-garlic mix, while those that received TMS consumed an average of $53 \mathrm{~g} / \mathrm{d}$ of salt.

\section{Discussion}

There were six survey days in 2016 (June 1, July 6, July 21, August 10, August 30, and September 13) while there were nine survey days in 2017 (May 29, June 7, June 23, July 7, July 13, July 28, August 17, August 30, and September 14). Fly survey in the first year for the RH group did not start until July 21 because the animals arrived late at the pasture. Similarly, the study surveys on the GO-N group started a week later in the second year. A limitation of the study was the lack of group replication. The fourth group that would have complemented the study design was not received. The third year of the trial to accommodate the limitation was not achieved.

Horn flies can be identified by their feeding behavior and resting locations on cattle (Tomberlin, 2010; Boxler, 2015). Even though confirmatory identification of all observed flies was not conducted, most of the observed pest flies rested on the crest-shoulder-rib areas, suggesting they were mainly horn flies. Identifying the fly species would require catching the flies (with sweep nets) directly from the grazing cows, but this was not practical due to inadequate handling facilities at the pasture site. Further, personal communication with cattle owners and the community pasture administrators confirmed that horn flies were the main pest flies in the region.

Management strategies that are practical and cost-effective while enhancing animals' productivity and welfare will increase the competitiveness of the beef industry. Determining the efficacy of supplementing grazing cows with garlic-infused supplements as a fly control strategy has received little research attention. Anecdotal accounts (from producers engaged in this feeding practice) about this supplementation strategy's effectiveness may not be reliable because of several factors. It is unlikely that the producers divided their stock into treatment groups (garlic-infused vs. control). Also, differences in the annual abundance of flies may confound subjective fly abundance assessments across different years. Therefore, this study's outcomes will provide objective evaluations while enabling producers to make feeding and purchasing decisions. 
Garlic powder contains an array of volatile sulfur-based bioactive compounds (Lawson and Gardner, 2005), which confer insectifugal and anti-oviposition properties (Thakur and Gupta, 2013). It is hypothesized that the strong odors emitted from the skin come from the organosulphur compounds metabolized after consuming garlic-based products. Because some scents are known to elicit sensitivity of the odor receptor neurons of insects (Maia and Moore, 2011), the volatile metabolites of garlic transported in the blood and emitted from the skin would deter the pest flies.

The average fly abundance during the survey dates showed some variation among the groups across the two years. Figure 1 showed an increasing number of pest flies starting in June, peaking in August, and declined in September as ambient temperatures dropped. Compared to the second year, the first year had fewer fly abundance peaks, probably due to fewer survey days as each peak may indicate different generations or life cycles of the pest flies.

The similarity in fly abundance between RH-N and RH-G shows the garlic treatment in the RH-G was not as efficacious for that group as the DM-G. The reasons behind this are unclear, given that DM-G showed fewer fly counts than the other groups. It is also uncertain whether the insectifugal effects observed in DM-G were due to innate (cattle breed composition) abilities. Steelman et al. (1997) had shown that fly abundance was related to differences in hair density typical of specific breeds. While some studies suggested that fly abundance could be related to coat color (Franks et al., 1964), others have shown the contrary (Ernst and Krafsur, 1984; Guglielmone et al., 2002). However, we minimized such confounding effects by analyzing data from crossbred cows with red coat color because several breeds or crossbreds show the red coat color. If breed differences were present among the various cow groups, the different fly abundance outcomes might be due to genotypexenvironment interactions. This assumption implies that using only coat color to identify or stratify animals may not be ideal for adjusting for breed representation in each treatment group. Accommodating differences due to breed composition could be executed using genomic breed composition results (Akanno et al., 2017). We recommend that future studies conduct a genomic breed composition before randomizing individuals to treatment groups.

The insectifugal outcomes of the $2 \%$ inclusion rate (especially with DM-G) are contrary to the reports of Bianchin et al. (1999), which reported that the inclusion rate did not reduce the population of horn flies in crossbred beef heifers. Massariol et al. (2011) did not find any difference when chopped garlic (mixed with concentrate) was fed to lactating Holstein cows at $100 \mathrm{~g}$ and $200 \mathrm{~g} / \mathrm{cow}$ per day. Chopping garlic may reduce the proportion of thiosulphinates produced due to incomplete enzymatic reaction (Amagase, 2006), suggesting that feeding chopped garlic (vs. powder) may reduce the amount of available bioactive compounds produced. It is also likely that the length of time (3 days) in that study was not enough to elicit the expected outcomes, in addition to the incomplete formation of the active compounds.

Treating grazing cows with other forms of garlic-based products have been shown to repel pest flies. The application of garlic-based sprays or pour-on effectively reduced fly abundance and defensive behaviors (Mohammed et al., 2016). Woolley et al. (2018) demonstrated that treating pastured dairy cows with topical garlic-based essential oil reduced fly densities, reduced fly-related defensive behavior, increased grazing and rumination time. They also reported that the treated cows traveled shorter distances than untreated cows. Topical applications will require routine animal handling, which may be intrusive on pastured cattle, thereby increasing production costs due to additional labor requirements. A free-choice feeding strategy is more attractive to producers due to less labor and less-frequent handling of grazing cattle.

This study did not show any differences between the treatment groups for salt intake after adjusting for group size differences. While the lack of differences between the two groups did not support anecdotal reports that adding garlic into supplements increased supplement consumption, the results did not account for the differences in body sizes that could drive supplement intake. There is considerable variability in individual salt intake in cattle (Reuter et al., 2017; Yelich et al., 2019). Therefore, additional studies are required to complement these results, where feed and mineral intakes and growth rates are measured for each animal. Future trials will be more informative if the bioactive compounds in each batch of supplements are measured to determine if such differences result in the observed outcomes. It is also unclear whether lacing other beef supplements (such as mineral supplements) with garlic powder or other products will have similar palatability or consumption outcomes as salt.

Finally, strategies that have the potential to improve productivity and profitability are important to beef producers. If validated, including garlic powder could be a more economical fly-control option for cattle producers. It is also a more attractive option because it does not require extra labor for producers with an existing mineral supplementation program. Further studies are necessary to understand other impacts on animal 
performance and health.

\section{Acknowledgements}

The Saskatchewan Ministry of Agriculture provided logistics support. Compass Minerals and Masterfeeds provided in-kind support of supplements. Dean Moore, Robert Holba and Glen Ockerman provided animal resources while Ed Sarazzin and Clarence Johnson managed the animals.

\section{References}

Akanno, E. C., Chen, L., Abo-Ismail, M. K., Crowley, J. J., Wang, Z., Li, C., .. Plastow, G. (2017). Genomic prediction of breed composition and heterosis effects in Angus, Charolais, and Hereford crosses using 50K genotypes. Canadian Journal of Animal Science, 97. 431-438. https://doi.org/10.1139/cjas-2016-0124

Amagase, H. (2006). Clarifying the real bioactive constituents of garlic. Journal of Nutrition, 136, 716-725. https://doi.org/10.1093/jn/136.3.716S

Bianchin, I., Gomes, A., Feijo, G. L. D., \& Vaz, E. C. (1999). Efficacy of garlic powder (Allium sativum) in the control of bovine parasites. Embrapa Beef Cattle Research Bulletin, 8, 31.

Boxler, D. J. (2015). The horn fly. NebGuide G1180. Retrieved from https://entomology.unl.edu/livestock/hornfly.pdf

Campbell, J. B. (1976). Effect of horn fly control on cows as expressed by increased weaning of calves. Journal of Economic Entomology, 69, 711-712. https://doi.org/10.1093/jee/69.6.711

Campbell, J. B., Berry, I. L., Boxler, D. J., Clanton, D. C., \& Deutscher, G. H. (1987). Effects of stable flies (Diptera: Muscidae) on weight gain and feed efficiency of feedlot cattle. Journal of Economic Entomology, 80, 117-119. https://doi.org/10.1093/jee/80.1.117

Canadian Council of Animal Care (CCAC). (2009). Guidelines on the care and use of farm animals in research, teaching, and testing. Retrieved from http://www.ccac.ca/Documents/Standards/Guidelines/Farm_Animals.pdf

Ernst, C. M., \& Krafsur, E. S. (1984). Horn fly (Diptera: muscidae): Sampling considerations of host breed and color. Environmental Entomology, 13, 892-894. https://doi.org/10.1093/ee/13.3.892

Franks, R. E., Burns, E. C., \& England, N. C. (1964). Color preference of the horn fly, Haematobia irritans, on beef cattle. Journal of Economic Entomology, 57, 371-372. https://doi.org/10.1093/jee/57.3.371

Guglielmone, A. A., Volpogni, M. M., Castro, H., Mangold, A. J., \& Anziani, O. S. (2002). A study of relative horn fly, Haematobia irritans (Diptera: Muscidae), abundance on Holstein steers and steers of two Holstein crosses. Veterinary Parasitology, 109, 141-5. https://doi.org/10.1016/S0304-4017(02)00255-8

Hashim, M. S., \& Davi, K. S. (2003). Insecticidal action of the polyphenolic rich fractions from the stem bark of Streblus asper on Dysdercus cingulalus. Fitoterapia, 8, 670-676. https://doi.org/10.1016/S0367-326X(03)00186-2

Haufe, W. O. (1982). Growth of range cattle protected from flies (Haematobia irritans) by ear tags impregnated with fenvalerate. Canadian Journal of Animal Science, 62, 567-573. https://doi.org/10.4141/cjas82-066

Karuppiah, P., \& Rajaram, S. (2012). Antibacterial effect of Allium sativum cloves and Zingiberofficinale rhizomes against multiple-drug resistant clinical pathogens. Asian Pacific Journal of Tropical Biomedicine, 2, 597-601. https://doi.org/10.1016/S2221-1691(12)60104-X

Lawson, L. D., \& Gardner, C. D. (2005). Composition, stability, and bioavailability of garlic products used in a clinical trial. Journal of Agricultural and Food Chemistry, 53, 6254-6261. https://doi.org/10.1021/jf050536+

Maia, M. F., \& Moore, S. J. (2011). Plant-based insect repellents: a review of their efficacy, development and testing. Malaria Journal, 10, S1-S11. https://doi.org/10.1186/1475-2875-10-S1-S11

Mathis, C. P., Cox, S. H., Löest, C. A., Petersen, M. K., Endecott, R. L., Encinias, A. M., \& Wenzel, J. C. (2008). Comparison of low-input pasture to high-input drylot backgrounding on performance and profitability of beef calves through harvest. The Professional Animal Scientist, 24, 169-174. https://doi.org/10.15232/S1080-7446(15)30832-9

Massariol, P. B., Olivo, C. J., Richards, N., Agnolin, C. A., Meinerz, G. R., Both, J. F., ... Martinelli, S. (2011). Ectoparasite load alteration in Holstein cows fed with different garlic (Allium sativum L.) levels. Revista Brasileira de Plantas Medicinais, 11, 37-42. http://dx.doi.org/10.1590/S1516-05722009000100007 
Mohammed, A. N., Abdel, A. N. M., \& Abdel, L. G. K. (2016). Field trial using combined treatment of garlic and organic sprayformula for flie's control and animal defensive behavior alleviation in cattle farm. Asian Journal of Animal Sciences, 10, 280-289. https://doi.org/10.3923/ajas.2016.280.289

Parra, L., Rojas, C., Catrileo, A., Galdames, R., Mutis, A., Birkett, M. A., \& Quiroz, A. (2013). Differences in the fly-load of Haematobia irritans (Diptera: Muscidae) on cattle is modified by endophyte infection of pastures. Electronic Journal of Biotechnology, 16, 4-4. https://doi.org/10.2225/vol16-issue5-fulltext-4

Reuter, R. R., Moffet, C. A., Horn, G. W., Zimmerman, S., \& Billars, M. (2017). Technical Note: daily variation in intake of a salt-limited supplement by grazing steers. The Professional Animal Scientist, 33, 372-377. https://doi.org/10.15232/pas.2016-01577

Steelman, C. D., Brown, M. A., Gbur, E. E., \& Tolley, G. (1997). The effects of hair density of beef cattle on Haematobia Irritans horn fly populations. Medical and Veterinary Entomology, 11, 257-264. https://doi.org/10.1111/j.1365-2915.1997.tb00404.x

Thakur, M., \& Gupta, D. (2013). Plant Extracts as oviposition deterrents against fruit flies, Bactrocera spp. infesting vegetable crops. Pesticide Research Journal, 25, 24-28.

Tomberlin, J. K. (2010) Protecting cattle from horn flies. Agrilife Extension, Texas A\&M system. Retrieved from https://agrilifecdn.tamu.edu/victoriacountyagnr/files/2010/07/Protecting-Cattle-from-Horn-Flies.pdf

Woolley, C. E., Lachance, S., DeVries, T. J., \& Bergeron, R. (2018). Behavioural and physiological responses to pest flies in pastured dairy cows treated with a natural repellent. Applied Animal Behaviour Science, 207, 1-7.

Yelich, J. V., Ellison, M. J., Hall, J. B., \& McGee, M. (2019). Intake behaviors of yearling steers grazing irrigated pasture and receiving either a free-choice salt-based mineral or a low-moisture molasses-based tub mineral, Translational Animal Science, 3, 749-760. https://doi.org/10.1093/tas/txz077

\section{Copyrights}

Copyright for this article is retained by the author(s), with first publication rights granted to the journal.

This is an open-access article distributed under the terms and conditions of the Creative Commons Attribution license (http://creativecommons.org/licenses/by/3.0/). 